\title{
A Clinical Analysis to Detect Risk Factors of Chronic Subdural Hematoma Recurrence
}

\author{
Ahmad Abdalla \\ Department of Neurosurgery, Assuit University, Assuit, Egypt \\ Email: pcahmadabdalla@yahoo.com
}

How to cite this paper: Abdalla, A. (2019) A Clinical Analysis to Detect Risk Factors of Chronic Subdural Hematoma Recurrence. Open Journal of Modern Neurosurgery, 9, 145-153.

https://doi.org/10.4236/ojmn.2019.92014

Received: January 6, 2019

Accepted: April 12, 2019

Published: April 15, 2019

Copyright $\odot 2019$ by author(s) and Scientific Research Publishing Inc. This work is licensed under the Creative Commons Attribution International License (CC BY 4.0).

http://creativecommons.org/licenses/by/4.0/ (c) (i) Open Access

\begin{abstract}
Introduction: The etiology of recurrence of chronic subdural hematoma (CSDH) after surgical evacuation has not been completely understood until now, but several risk factors for recurrence have been reported. Meanwhile, the definitive risk factors have not been defined until now. Aim of Study: Analyze the potential risk factors, and emphasize preoperative, operative and post-operative ones for CSDH recurrence. Patients and Methods: This study is a prospective randomized clinical trial study including 82 patients with symptomatizing CSDH who underwent burr holes procedure and irrigation with closed system drainage for CSDH at Neurosurgical Department-Assuit University Hospital from July 2016 to July 2018. The possible factor studied for recurrence included age, hypertension, diabetes mellitus, liver diseases, Hemoglobin $(\mathrm{Hb})$ level, prothrombin concentration and time (PC and PT), hematoma thickness and internal architecture, number of burr holes and position of drain, duration of drainage, amount of drainage and presence of postoperative residual hematoma on follow up CT brain. Multiple logistic regression analysis is used to assess the predictors of recurrence. Results: This study included 70 males and 12 females. The mean age was 58.9 years (range 34 - 93 years). 6 patients presented with recurrent CSDH. Age above 60 years, Hypertension, Diabetes mellitus (DM), prolong PT, separated and trabecular internal architecture of hematoma, and the thickness of hematoma more than $20 \mathrm{~mm}$ in pre-operative CT and postoperative residual hematoma were statistically significant factors for recurrence of CSDH. Conclusion: This information might be helpful in detecting patients with possible high incidence of recurrence and directing for close follow ups and acts that may reduce the incidence of recurrence.
\end{abstract}

\section{Keywords}

Chronic Subdural Hematoma, Risk Factors, Recurrence 


\section{Introduction}

Chronic subdural hematoma (CSDH) is a common disease in neurosurgical practice and the incidence is increasing. The initial bleeding in CSDH is likely to have a venous origin. Blood evokes an inflammatory reaction resulting in fibrin deposition, followed by organization and formation of neomembranes. A high plasminogen content within the outer neomembrane enhances fibrolytic activity. This results in effusions of plasma or rebleeding from the neomembranes into a subdural collection. In the last twenty years, the most frequently used surgical techniques have been burr-hole drainage, irrigation and closed drainage. However it has been reported that recurrence rate ranges from $3.7 \%$ to $30 \%$ [1].

The etiology of recurrence of CSDH has not been completely understood until now, but several risk factors for recurrence of $\mathrm{CSDH}$ have been reported, including advanced age, brain atrophy, bilateral CSDH, hematoma density, seizures, diabetes mellitus, bleeding tendency, alcohol abuse, and postoperative posture [2]. However, the definitive risk factors have not been defined until now. Most studies focused on the preoperative factors, but few concerned the surgical or postoperative factors.

\section{The Aim of the Study}

The aim of the study was to analyze the potential risk factors, nd emphasize preoperative, operative and post-operative ones for $\mathrm{CSDH}$ recurrence.

\section{Patients and Method}

\subsection{Study Design}

This study is a prospective randomized hospital-based clinical trial study including total of 82 patients with CSDH who were admitted to Neurosurgical Department-Assuit University Hospital from July 2016 to July 2018 and underwent burr holes procedure and irrigation with closed system drainage.

All patients were subjected to detailed history, general clinical examination, complete neurological examination included GCS and motor power, laboratory investigation as $\mathrm{cbc}, \mathrm{pc} \& \mathrm{pt}$, liver function tests and computerized tomogram (CT) of the brain.

\subsection{Operative Technique}

Under general endotracheal anaesthesia, the patient was positioned in supine position with the head slightly elevated $\left(15^{\circ}\right)$ and tilted to healthy side.

After scalp incision and burr hole/holes craniostomy was/were done, the outer layer of dura cauterized by bipolar diathermy, then we opened the dura in criss-cross manner with gradual evacuation of hematoma. The margin of opened dura was cauterized until shrunken completely to avoid bleeding from the opened dura to the subdural space. after that genital irrigation of hematoma by normal saline until complete evacuation of hematoma and the wash comes out clear, closed system drainage with negative pressure was applied either subdu- 
rally or subgaleally. Finally the wound closed in layers. Patient admitted to the intensive care unit for 24 hours before referred to ward unless the patient deteriorated. The patient was given I.V antibiotic (usually third generation of cephalosporine), antiepileptic and analgesic, in addition the patient was encouraged for fluid intake and I.V fluid for 24 hours. The patient was allowed to sit and walk in the next day. The drainage system was removed when drainage stopped mostly by the end of next day. The patient discharged from hospital after 2 - 3 day and scalp stiches were removed 10 days post-operation.

\subsection{Outcomes Assessment}

The Glasgow coma scale (GCS) and motor power were used to assist patient clinical outcome. Also postoperative CT image was done within 48 to 72 hours postoperatively to detect any residual hematoma. Once the patient clinically and radiologically improved, discharged and followed in an outpatient clinic bases.

Numerous factors influencing recurrence have been reported in the literature. The risk factors that will be studied for recurrence in our study are:

\section{- Preoperative risk factors:}

1) Age of patient.

2) Associated chronic diseases like hypertension, diabetes mellitus, and liver diseases.

3) Laboratory investigation like Hb level, PC and PT.

4) Preoperative radiological parameters:

a) Hematoma thickness: less than $10 \mathrm{~mm}$, from $10 \mathrm{~mm}$ to $20 \mathrm{~mm}$ or more than $20 \mathrm{~mm}$.

b) Internal architecture of CSDH according to Nakaguchi et al. [3] as homogenous, laminar, separated and trabecular.

\section{- Operative risk factors:}

1) Number of burr hole: one or two burr holes.

2) Position of drain: subgaleal or subdural.

\section{- Postoperative risk factors:}

1) Duration of drainage: less than 3 days and 3 - 5 days.

2) Amount of drainage: <100 cc, $(100-200 \mathrm{cc}),(200-300 \mathrm{cc})$, and greater than $300 \mathrm{cc}$.

3) Present of postoperative residual hematoma on follow up CT brain.

\section{Statistical Analysis}

The data was tested for normality using the Anderson-Darling test and for homogeneity variances prior to further statistical analysis. Categorical variables were described by number and percentage (No., \%), where continuous variables described by mean and standard deviation (Mean, SD). Chi-square test and fisher exact test were used to compare between categorical variables. Multiple logistic regression analysis was used to assess the predictors of recurrence. A two-tailed $p<0.05$ was considered statistically significant. The whole analysis was per- 
formed with the IBM SPSS 20.0 software.

\section{Results}

This study included 70 patients (85.4\%) males and 12 patients (24.6\%) females. The mean age was 58.9 years (range 34 - 93 years). 65 patients (79.2\%) presented with headache and hemiparesis, while 17 patients $(20.8 \%)$ had disturbed conscious level with GCS ranged from 8 - 14. Postoperatively, all patients showed marked clinical improvement regarding conscious level and motor power. Six patients (7.3\%) presented with recurrence of CSDH as shown in Table 1. Allthese patients underwent re-evacuation with good outcome. Table 2 summarized the results of the risk factors for reccurence.

* Results of preoperative risk factors for recurrence:

All patients with recurrence of CSDH were above 60 years old which was not statistically significant $(\mathrm{p}=0.246)$.

Diabetes and hypertension were statistically significant factors regarding recurrence of CSDH; 4 out of 6 recurrent patients were diabetic (66.7\%) with ( $\mathrm{p}=$ $0.027)$, while all patients with recurrence were hypertensive $(100 \%)(\mathrm{P}=0.021)$. There is no relationship between recurrent $\mathrm{CSDH}$ and patients with chronic liver diseases $(\mathrm{p}=0.889)$.

Among recurrence patients, about 2 patients (33.3\%) had prothrombin time more than 14 seconds (control 11.5 - 12 second) with significant correlation with recurrence $(\mathrm{p}=0.025)$, while prothrombin concentration of more than $70 \%$ is a protective factor of recurrence CSDH $(\mathrm{p}=0.029)$.

The statistically significant recurrence occurred in 2 patients (33.3\%) and 4 patients $(66.7 \%)$ with both separated and trabecular hematoma respectively; the $\mathrm{p}$-values were $(\mathrm{p}=0.044)$ and $(\mathrm{p}=0.025)$ respectively while there were no recurrence among others.

All six patients displayed recurrence had radiological hematoma thickness more than $20 \mathrm{~mm}$ and was statistically insignificant $(\mathrm{p}=0.34)$.

* Results of operative risk factors for recurrence:

All the recurrent cases of CSDH occurred exclusively among patients underwent two burr holes $\mathrm{n}=6(100 \%)$ and also recurrence was more among patients with subgalial $(\mathrm{Sg})$ drain $\mathrm{n}=4$ out of $6(66.7 \%)$ in comparison with patients with subdural ( $\mathrm{Sd}$ ) drain but that was statistically insignificant where $\mathrm{p}$-value was ( $\mathrm{p}=$ $0.633)$ and $(\mathrm{p}=0.599)$ respectively.

* Results of postoperative risk factors for recurrence:

Although recurrence was exclusively among patients with the drain maintained for 3 days or more, it is statistically insignificant $(\mathrm{p}=0.52)$. Also there

Table 1. Frequency distribution of studied patients as regard postoperative recurrence.

\begin{tabular}{llcc}
\hline & & No. & $\%$ \\
\hline$\bullet \quad$ Recurrence & 6 & 7.3 \\
$\bullet \quad$ Non-recurrence & 76 & 92.7
\end{tabular}


Table 2. Results of prognostic risk factors for recurrence ( $p$ value $<0.05^{*}$ is statistically significant).

\begin{tabular}{|c|c|c|c|c|}
\hline Risk factors & $\begin{array}{c}\text { No. of } \\
\text { patients }\end{array}$ & $\begin{array}{c}\% \\
\text { percentage }\end{array}$ & $\begin{array}{c}\text { Recurrent } \\
\text { patients }\end{array}$ & $\mathrm{p}$ value \\
\hline - Age & & & & \\
\hline - $\quad<40$ & 4 & 4.9 & - & \\
\hline - $40-50$ & 8 & 9.7 & - & \\
\hline - $50-60$ & 20 & 24.4 & - & \\
\hline - $\quad>60$ & 50 & 61 & 6 & 0.246 \\
\hline - $\quad$ Diabetes mellitus (D.M) & 20 & 24.4 & 4 & $0.027^{*}$ \\
\hline - Hypertension & 28 & 34.1 & 6 & $0.021^{\star}$ \\
\hline - Liver disease & 4 & 4.9 & - & \\
\hline - Prothrombin time $>14 \mathrm{sec}$. & 6 & 7.3 & 2 & $0.025^{\star}$ \\
\hline - $\quad$ Prothrombin Conc. $<70 \%$ & 6 & 7.3 & 2 & $0.029^{*}$ \\
\hline - $\quad$ Hb level $<10 \mathrm{mg} / \mathrm{Dl}$ & 6 & 7.3 & - & \\
\hline - Internal architecture of SDH: & & & & \\
\hline - Homogenous & 42 & 51.2 & - & \\
\hline - Laminar & 14 & 17.1 & - & \\
\hline - Separated & 10 & 12.2 & 2 & $0.044^{\star}$ \\
\hline - Trabecular & 16 & 19.5 & 4 & $0.025^{*}$ \\
\hline - SD hematoma thickness: & & & & \\
\hline - $\quad 10-20 \mathrm{~mm}$ & 14 & 17.1 & - & \\
\hline - $\quad>20 \mathrm{~mm}$ & 68 & 82.9 & 6 & $0.034^{\star}$ \\
\hline - Number of burr-holes: & & & & \\
\hline - 1 burr-hole & 10 & 12.2 & - & \\
\hline - 2 burr holes & 72 & 87.8 & 6 & 0.633 \\
\hline - Position of drain: & & & & \\
\hline - Subgleal (Sg) & 62 & 75.6 & 4 & \\
\hline - Subdural (Sd) & 20 & 24.4 & 2 & 0.599 \\
\hline - Duration of drainage: & & & & \\
\hline - Less than 3 days & 28 & 34.1 & - & \\
\hline - 3 days or more & 54 & 65.9 & 6 & 0.052 \\
\hline - Amount of drainage: & & & & \\
\hline - Less than $100 \mathrm{cc}$ & 2 & 2.4 & - & \\
\hline - $100 \mathrm{cc}-$ & 36 & 43.9 & 4 & 0.821 \\
\hline - $200 \mathrm{cc}-$ & 32 & 39 & 2 & 0.596 \\
\hline - More than $300 \mathrm{cc}$ & 12 & 14.6 & - & \\
\hline $\begin{array}{l}\text { Postoperative residual hematoma on } \\
\text { follow up CT Brain }\end{array}$ & 9 & 10.9 & 4 & $0.005^{*}$ \\
\hline
\end{tabular}

were no correlation between recurrence and amount of drainage ( $\mathrm{p}>0.596)$.

Out of all patients of recurrence, about 4 patients $(66.7 \%)$ had postoperative residual hematoma and was statistically significant $(\mathrm{p}<0.001)$.

\section{Discussion}

The literature states that the risk of CSDH recurrence increases with age. This may be correlated to brain atrophy and decay of cerebral veins. In our study, no 
significant difference was observed between the average ages of the patients and the incidence of recurrence and non-recurrent, this may be attributed to the small sample size. These results are consistent with those of Stasnistic et al. [4].

Also, no significant difference was observed between the incidence of recurrence of CSDH among patients with liver disease and those who had no liver disease, which may be attributed to inactive chronic hepatitis while the liver is not in cirrhotic stage. On the contrary patients in Gronbaek et al. [5] were in cirrhotic stage of liver disease and this was associated with increased risk of recurrence.

Also in this study prolonged prothrombin time was correlated with increased recurrence of $\mathrm{CSDH}$, due to deficiency of clotting factors (I, II, V, VII, and X) made by the liver.

We found that hypertension was a risk factor of recurrence, as it may cause damage to blood vessels specially in elderly patients whose blood vessels were sclerotic and inelastic so these vessels are more liable to rupture, in contrary with the study of Torihashit et al. [6] which showed that hypertension did not have any effect on recurrence.

Regarding diabetes, Torihashi et al. [6], speculated that viscosity increases in diabetic patients, an osmotic pressure increase will trigger coagulation and decrease the risk of hematoma; however, they could not determine diabetes as an effective factor in their studies. Tugcu et al. found that was no association between diabetes and CSDH recurrence [7]. In our study, diabetes was a significant risk factor of recurrence of CSDH, which may be correlated to capillary vasculopathy, particularly in patients with $\mathrm{CSDH}$, where there is a sufficient capillary network on the outer membrane and the vasculopathy here may lead to a growth or recurrence of the hematoma.

Regarding the internal architecture of CSDH: separated stage of CSDH is consideedr as a significant risk factor of CSDH in many reports [4] [5] [7] [8] [9] [10], others stated that the laminar stage of CSDH is a risk factor of recurrence [1] and homogenous stage has the lowest recurrence rate in all previous reports. In this study we found that both separated and trabecular hematomas are risk factors of recurrence, due to hyperfibrinolysis and the rebleeding from the neomembrane (the blood vessles of neomembrane is premature and friable) is high, so the operation of evacuation of $\mathrm{CSDH}$ is preferable to do in homogenous stage to decrease the incidence of recurrence unless severe symptoms are present.

Many authors stated that hematoma thickness $>20 \mathrm{~mm}$ was significant risk factor of recurrence CSDH [1] [6] [8] [10] [11], the pathogenesis is unknown but it has been stated that any factor leading to a prolonged postoperative widening of the hematoma cavity (brain atrophy, residual hematoma, massive subdural air collection, excessive fluid drainage through subdurally placed drains) may cause an impaired adhesion between the inner and outer hematoma neomembranes and thus facilitates postoperative recurrence [12] [13].

Few studies have been conducted to compare directly the use of one and two burr-hole techniques for the surgical management of chronic subdural hemato- 
ma. Despite this, both techniques are widely used by neurosurgeons [14], and no consensus has been reached whether one technique is superior to the other.

Hammer et al. [13] Oh et al. [15] reported no significant difference in recurrence rate observed between $2 \mathrm{BHC}$ (two burr holes craniostomy) and $1 \mathrm{BHC}$ (one burr hole craniostomy), but Taussky et al. [16] reported a significantly higher rate of recurrence was found for $1 \mathrm{BHC}$ compared to $2 \mathrm{BHC}$. In our study, recurrence rate was more in patient underwent $2 \mathrm{BHC}$ but that was statistically insignificant.

We did not find studies that investigated directly the comparison between subdural with subgaleal drainage and incidence of recurrence of CSDH. Although some reported that the recurrence rate was low when using subdural (Sd) drainage [8], there is still a disagreement on whether subdural drainage should be performed postoperatively. Placement of a subgaleal ( $\mathrm{Sg}$ ) drain after a craniotomy has been reported with a low recurrence rate in the thesis of Gazzeri et al. [17]. In this study although recurrence rate was higher in patients with subgalial (Sg) drainage compared to patients with subdural (Sd) drainage but that was statistically insignificant.

Regarding the relationship between volume of drainage and recurrence rate: Hammar et al. [13] reported that there is a correlation between postoperative drainage volume with the recurrence rate, when the total drainage volume was below $200 \mathrm{ml}$, the recurrence rate increased from $0 \%$ to $6.4 \%$, while Cheng et al. [1] found that larger drainage amount is independently associated with higher recurrence rate of CSDH. They suggested some explanations. First, some CSDH with large post-operative drainage amount may be transformed from subdural hygroma, notably it has been reported that persistent subdural hygroma is an important risk factor in the development of CSDH. Second, some elderly patients had severe cerebral atrophy and poor brain re-expansion, after hematoma evacuation, cerebrospinal fluid may pass through into the low pressure subdural space. If there is a large amount of subdural effusion, the bridge veins in the subdrual space may tear gradually and bleeding from the inner membrane or more anti-thrombolytic factors produced made the CSDH recurrent. However, Stanisic et al. [4] reported that there was no correlation between postoperative drainage volume and the recurrence rate, which correlate with our results, where no relationship between recurrence rate and volume of drainage was observed.

Stanistic et al. [4] reported that the appearance of acute subdural clots in cranial base CSDHs on CT scans obtained within four days post surgery had a higher recurrence rate than those with absence of these clots. Kong et al. [18] suggested that recurrence rate depends on the removal of the residual semisolid subdural hematoma component and the removal, dilution and inactivation of endogenous fibrinolytic agents and refilling the subdural cavity with saline prevent the influx of air into the subdural space which will reduce the recurrence rate of $\mathrm{CSDH}$.

In our study, the recurrence was significant in patients with postoperative residual hematoma followed up by brain CT 24 to 48 hours and within 3 weeks af- 
ter surgery, so it is important to remove solid or semi-solid hematoma either by continuous irrigation or craniectomy.

\section{Conclusions}

Certain risk factors influencing postoperative recurrence of $\mathrm{CSDH}$ were articulated in our study as Hypertension, DM, prolonged PT, separated and trabecular internal architecture of hematoma, and the thickness of hematoma more than 20 $\mathrm{mm}$ in pre-operative CT and postoperative residual hematoma. However, there was no correlation between age of patient, inactive chronic liver disease, number of burr hole, position of drain and amount of drainage and recurrence of CSDH.

This information might be helpful for identifying patients with high incidence of recurrence in order to set close follow ups and acts to reduce the recurrence such as evacuation of hematoma which is performed at homogenous stage and complete evacuation of hematoma may reduce the incidence of recurrence.

\section{Limitation of the Study and Recommendation}

Limitation of our study was the small sample size and short duration, so further studies should be conducted over longer periods and large number of patient to confirm the risk factors of $\mathrm{CSDH}$ recurrence.

\section{Conflicts of Interest}

The author declares no conflicts of interest regarding the publication of this paper.

\section{References}

[1] Cheng, C., et al. (2014) Radiological Features and Post-Operative Drainage amount Independently Predict Recurrence of Chronic Subdural Hematoma after Burr-Hole. Journal of Neurological Disorders, 2, 148.

[2] Lee, J.M., Park, J.C. and Kim, J.H. (2016) Retrospective Analysis of Risk Factors for Recurrent Chronic Subdural Hematoma. The Nerve, 2, 54-58. https://doi.org/10.21129/nerve.2016.2.2.54

[3] Nakaguchi, H., Tanishima, T. and Yoshimasu, N. (2001) Factors in the Natural History of Chronic Subdural Hematomas That Influence Their Postoperative Recurrence. Journal of Neurosurgery, 95, 256-262. https://doi.org/10.3171/jns.2001.95.2.0256

[4] Stanisic, M. and Mahesparan, R. (2005) Clinical Article Treatment of Chronic Subdural Hematoma by Burr-Hole Craniostomy in Adults: Influence of Some Factors on Postoperative Recurrence. Acta Neurochirugica, 147, 1249-1257. https://doi.org/10.1007/s00701-005-0616-1

[5] Gronbaek, H., et al. (2008) Liver Cirrhosis, Other Liver Diseases, and Risk of Hospitalisation for Intracerebral Haemorrhage: A Danish Population-Based Case-Control Study. BMC Gastroenterology, 8, 16. https://doi.org/10.1186/1471-230X-8-16

[6] Torihashi, K., et al. (2008) Independent Predictors for Recurrence of Chronic Subdural Hematoma: A Review of 343 Consecutive Surgical Cases. Neurosurgery, 63, 1125-1129. https://doi.org/10.1227/01.NEU.0000335782.60059.17 
[7] Tugcu, B., et al. (2010) Can Recurrent Chronic Subdural Hematomas Be Predicted? A Retrospective Analysis of 136 Cases. Dusunen Adam, 23, 44-49.

[8] Ko, B.-S., et al. (2008) Clinical Analysis of Risk Factors Related to Recurrent Chronic Subdural Hematoma. Journal of Korean Neurosurgical Society, 43, 11-15. https://doi.org/10.3340/jkns.2008.43.1.11

[9] Huang, Y., Lin, W., Lu, C. and Chen, W. (2014) Volume of Chronic Subdural Haematoma: Is It One of the Radiographic Factors Related to Recurrence? Injury, 45, 1327-1331. https://doi.org/10.1016/j.injury.2014.02.023

[10] Jung, Y.-G., Jung, N.-Y. and Kim, E. (2012) Independent Predictors for Recurrence of Chronic Subdural Hematoma. Acta Neurochirurgica, 154, 1541-1548. https://doi.org/10.1007/s00701-012-1399-9

[11] Matsumoto, K., et al. (1999) Recurrence Factors for Chronic Subdural Hematomas after Burr-Hole Craniostomy and Closed System Drainage. Neurological Research, 21, 277-280. https://doi.org/10.1080/01616412.1999.11740931

[12] Ohba, S., Kinoshita, Y. and Nakagawa, T. (2013) The Risk Factors for Recurrence of Chronic Subdural Hematoma. Neurosurgical Review, 36,145-150.

https://doi.org/10.1007/s10143-012-0396-Z

[13] Hammer, A., et al. (2016) Predictors for Recurrence of Chronic Subdural Hematoma. Turkish Neurosurgery, 1-7. https://doi.org/10.5137/1019-5149.JTN.17347-16.1

[14] Nakaguchi, H., Tanishima, T. and Yoshimasu, N. (2000) Relationship between Drainage Catheter Location and Postoperative Recurrence of Chronic Subdural Hematoma after Burr-Hole Irrigation and Closed-System Drainage. Journal of Neurosurgery, 93, 791-795. https://doi.org/10.3171/jns.2000.93.5.0791

[15] Oh, H.-J., et al. (2010) Postoperative Course and Recurrence of Chronic Subdural Hematoma. Journal of Korean Neurosurgical Society, 48, 518-523. https://doi.org/10.3340/jkns.2010.48.6.518

[16] Taussky, P., Fandino, J. and Landolt, H. (2008) Number of Burr Holes as Independent Predictor of Postoperative Recurrence in Chronic Subdural Haematoma. British Journal of Neurosurgery, 22, 279-282. https://doi.org/10.1080/02688690701818885

[17] Gazzeri, R., et al. (2007) Continuous Subgaleal Suction Drainage for the Treatment of Chronic Subdural Haematoma. Acta Neurochirurgica, 149, 487-493. https://doi.org/10.1007/s00701-007-1139-8

[18] Kong, W., Kim, B., Cho, K. and Hong, S. (2012) Factors Affecting Postoperative Recurrence of Chronic Subdural Hematoma. Korean Journal of Neurotrauma, 8 , 122-127. https://doi.org/10.13004/kjnt.2012.8.2.122 